\title{
Implementing and testing theoretical fission fragment yields in a Hauser-Feshbach statistical decay framework
}

\author{
Patrick Jaffke $^{1, \star}$, Peter Möller ${ }^{1}$, Ionel Stetcu ${ }^{1}$, Patrick Talou ${ }^{1}$, and Christelle Schmitt ${ }^{2,3}$ \\ ${ }^{1}$ Theoretical Division, Los Alamos National Laboratory, Los Alamos NM, 87545, USA \\ ${ }^{2}$ Grand Accélérateur National d'lons Lourds, CEA/DSM-CNRS/IN2P3, Caen, France \\ ${ }^{3}$ Institut Pluridisciplinaire Hubert Curien, 23 rue du Loess, BP. 28, 67037 Strasbourg Cedex 2, France
}

\begin{abstract}
We implement fission fragment yields, calculated using Brownian shapemotion on a macroscopic-microscopic potential energy surface in six dimensions, into the Hauser-Feshbach statistical decay code CGMF. This combination allows us to test the impact of utilizing theoretically-calculated fission fragment yields on the subsequent prompt neutron and $\gamma$-ray emission. We draw connections between the fragment yields and the total kinetic energy TKE of the fission fragments and demonstrate that the use of calculated yields can introduce a difference in the $\langle\mathrm{TKE}\rangle$ and, thus, the prompt neutron multiplicity $\bar{v}$, as compared with experimental fragment yields. We deduce the uncertainty on the $\langle\mathrm{TKE}\rangle$ and $\bar{v}$ from this procedure and identify possible applications.
\end{abstract}

\section{Introduction}

Recent advances in theoretical models and computational abilities have allowed one to calculate many fission fragment properties at scission. Several models exist: macroscopic nuclear shape models [13], self-consistent microscopic models using effective two-body nuclear forces [4-6], or macroscopicmicroscopic models of the potential energy surfaces with various treatments of the dynamics on these surfaces, such as Brownian shape motion or hydrodynamical models of the inertia and friction tensors in the equations of motion [7-9]. All of these can be used to determine fission fragment yields in charge $Z$, or mass $A$, or both. Certain models are capable of calculating the total kinetic energy TKE of the fission fragments and even the nuclear deformation at scission as well. These methods become powerful tools towards a predictive model of fission, where one could calculate the scission configurations for compound nuclei that are experimentally impossible to measure.

Combining theoretical fragment yields with a Hauser-Feshbach [10] statistical decay framework, such as CGMF [11], provides one with a method to evaluate the impact of using these calculated fragment yields instead of experimental ones on the prompt neutron and $\gamma$-ray data. Primary fission fragment data already provide a direct comparison with calculated yields, but the addition of prompt neutron and $\gamma$-ray data strengthens the constraints on the theoretical yields models. It is this secondary step we illustrate here for the fission reactions ${ }^{239} \mathrm{Pu}(\mathrm{n}, \mathrm{f})$ and ${ }^{241} \mathrm{Pu}(\mathrm{n}, \mathrm{f})$ with neutron energies $E_{n}$ from thermal to $10 \mathrm{MeV}$. These cases are used to estimate the uncertainties in using theoretical yields for plutonium isotopes in the range ${ }^{236-244} \mathrm{Pu}$.

^e-mail: pjaffke@lanl.gov 
Our process is as follows: we first detail the necessary input for the Hauser-Feshbach calculations, including our average total kinetic energy $\langle\mathrm{TKE}\rangle$ parameterization, the assumed spin distribution and its dependence on $E_{n}$, the multi-chance fission probabilities, and the distributions of TKE parameters with fragment mass. Next, we present a simple comparison of fragment yields for ${ }^{239} \mathrm{Pu}(\mathrm{n}, \mathrm{f})$ for which we have experimental data at various incident neutron energies [12]. Finally, we conduct the full CGMF calculations to determine the sensitivity of fission observables to the use of theoretically-calculated fission fragment yields, as compared with the experimental ones.

\section{Input}

The fission fragment yields are calculated from a macroscopic-microscopic (macro-micro) model that uses six shape parameters to determine $Y(Z, A)$ [13]. Previous studies have shown good agreement between the calculated charge yields and the experimental ones for a variety of compound nuclei and incident neutron energies [7, 14]. In our CGMF calculations, we take the mass yields from the macro-micro model and the charge distribution follows that of Wahl [15]. One could use the charge distributions directly from the calculated $Y(Z, A)$, but this would require an increase in computation time to achieve reasonable statistics in the wings of the charge distributions. As the calculated charge distributions are similar to those of Wahl, we use the systematics instead. The $\langle\mathrm{TKE}\rangle$ and its dependence on mass $\langle\mathrm{TKE}\rangle(A)$ are taken from experimental data. For example, at thermal energies values of $177.76 \mathrm{MeV}$ [16-18] and $178.89 \mathrm{MeV}$ [19-21] are used for ${ }^{239} \mathrm{Pu}(\mathrm{n}, \mathrm{f})$ and ${ }^{241} \mathrm{Pu}(\mathrm{n}, \mathrm{f})$, respectively. The slopes $\partial\langle\mathrm{TKE}\rangle / \partial E_{n}$ are taken from a variety of sources $[12,19,22]$. The $\langle\mathrm{TKE}\rangle(A)$ are taken from Vorobyeva [23, 24] for the various plutonium isotopes, but we have also used other sources [2527] and found minimal impact on our results. The sampled TKE for each event follows a Gaussian centered about $\langle\mathrm{TKE}\rangle(A)$ with a variance $\sigma_{\mathrm{TKE}}^{2}(A)$, taken from Ref. [18, 28]. For isotopes that did not have data on the distribution parameters, $\langle\mathrm{TKE}\rangle(A)$ and $\sigma_{\mathrm{TKE}}^{2}(A)$, we take the data of the nearest available plutonium isotope and allow for an overall scaling in magnitude. We determine the $\langle\mathrm{TKE}\rangle$ for plutonium isotopes without data with a local $Z^{2} / A^{1 / 3}$ fit, similar to the Viola systematics [29]. We note that this simple two-point fit of the above data is quite inadequate, but could be improved with additional data. When no data is available on the slope $\partial\langle\mathrm{TKE}\rangle / \partial E_{n}$, we use the average over all plutonium isotopes with data.

The spin distributions of the fragments follows that of previous papers [30, 31] and the average spin $\langle J\rangle$ has an exponential form with $E_{n}$. The value at thermal neutron energies is fitted such that average prompt neutron multiplicity $\bar{v}$ matches the experimental data, when available, and the asymptote is derived from fits to ${ }^{238} \mathrm{U}(\mathrm{n}, \mathrm{f})$ prompt $\gamma$-ray multiplicities.

Multi-chance fission probabilities are calculated with the $\mathrm{CoH}$ code [32]. We note that this parameter is highly dependent on the fission barrier heights and level density parameters, which are not experimentally bound very well. We take fission barrier heights and curvatures from JENDL-4.0 [33]. Our procedure is to fit the total fission cross-section from ENDF/B-VII.1 [34] with the above barrier heights and curvatures. The level density parameters are adjusted to obtain the best fit and we use the resulting multi-chance probabilities.

With the above prescription, the only remaining input are the fission fragment yields themselves. We fit the theoretically predicted mass yields with 3 Gaussians. The parameters of the Gaussians, weight $w$, mean $\mu$, and variance $\sigma^{2}$, are dependent on the incident neutron energy $E_{n}$. This is done to provide a smooth function of the mass yields at any $E_{n}$, rather than an interpolation of tabulated values. They have the following form:

$$
w_{i}\left(E_{n}\right)=\frac{w_{i}}{1+e^{\left(E_{n}-x_{i}\right) / y_{i}}}, \quad \mu_{i}\left(E_{n}\right)=m_{i} E_{n}^{2}+n_{i} E_{n}+p_{i}, \quad \sigma_{i}\left(E_{n}\right)=s_{i} E_{n}^{2}+t_{i} E_{n}+r_{i}
$$


The weights of the Gaussians follow a Fermi-function with $E_{n}$ and the variances and means are allowed to be quadratic in $E_{n}$. Obviously, the mean for the symmetric Gaussian is $\mu_{3}=A_{C} / 2$, where $A_{C}$ is the mass of the fissioning nucleus, and one of the weights can be constrained via $2 w_{1}+2 w_{2}+w_{3}=2$, reducing the number of fitted parameters. We have found that using a linear function for $\sigma_{i}\left(E_{n}\right)$, instead of the quadratic one in Eq. (1), produces very good fits as well. The 3 Gaussian fit smoothly transitions to higher excitation energies and when multi-chance fission occurs, the proper compound nucleus' Gaussian parameters are selected, as we have calculated yields for ${ }^{236-244} \mathrm{Pu}$, instead of a shift in the means [35].

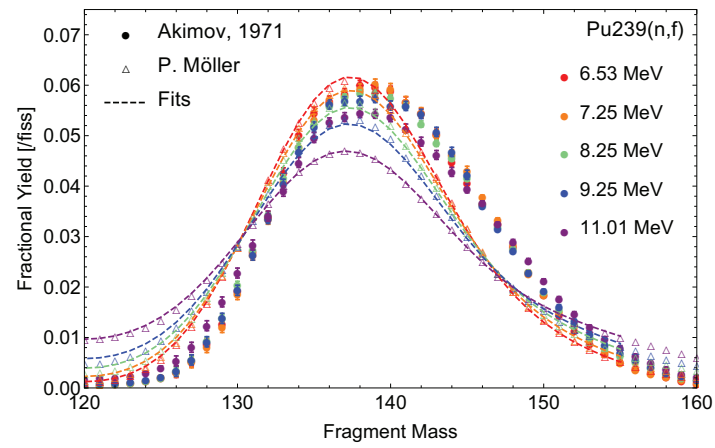

Figure 1. Comparison of the mass yields for the neutron-induced fission of ${ }^{239} \mathrm{Pu}$ from Ref. [12] (circles) and the calculated yields via Ref. [13] (triangles). Listed are the excitation energies, assuming a binding energy of $E_{b}=6.53 \mathrm{MeV}$ for ${ }^{239} \mathrm{Pu}$. Thus, the $6.53 \mathrm{MeV}$ (red) symbols and curves denote thermal fission. The dashed lines are the 3 Gaussian fits. We have included a $4.5 \mathrm{amu}$ mass resolution in the calculated yields. While none of the listed excitation energies in this plot are above second-chance fission, we included this effect in our study.

The mass yields of Ref. [13] are compared with the data of Ref. [12] at a variety of excitation energies. We have applied a mass resolution of 4.5 amu to the theoretical yields. One can see in Fig. 1 that there appears to be a shift in average heavy fragment mass $\left\langle A_{h}\right\rangle$ and the calculated yields appear to show a more rapid increase of the symmetric region with excitation energy. Overall, the calculated yields reproduce the general trends of the data, but the under-prediction of the mass yields around $A \sim 145$ causes a shift in $\left\langle A_{h}\right\rangle$ of about 1.15 amu relative to data. The impact of the excitation energy on the symmetric region is also more subtle in Akimov. The effect of these differences on the prompt particle emission is studied next.

\section{Prompt particle emission}

We perform Hauser-Feshbach fission fragment decay calculations for neutron-induced fission reactions on various plutonium isotopes. Most instructive is the case of ${ }^{239} \mathrm{Pu}(\mathrm{n}, \mathrm{f})$ as it contains experimental data on the primary fragment yields over a range of incident energies. We also show the results for ${ }^{241} \mathrm{Pu}(\mathrm{n}, \mathrm{f})$, where we only have the theoretical yields. In Fig. 2, we illustrate the total prompt neutron multiplicity as a function of incident neutron energy, which we note includes prescission neutrons. The comparison shows three data sources [34, 36, 37] and three computed cases. Case $A$ corresponds to a calculation that uses the $Y(A)$ from Ref. [12] and no adjustments have been made to the TKE input parameters described above. Case $B$ swaps out the experimental $Y(A)$ with the calculated values and maintains the same TKE input parameters as case $A$. Case $D$ adjusts the TKE parameters so as to match the $\bar{v}\left(E_{n}\right)$ data. 

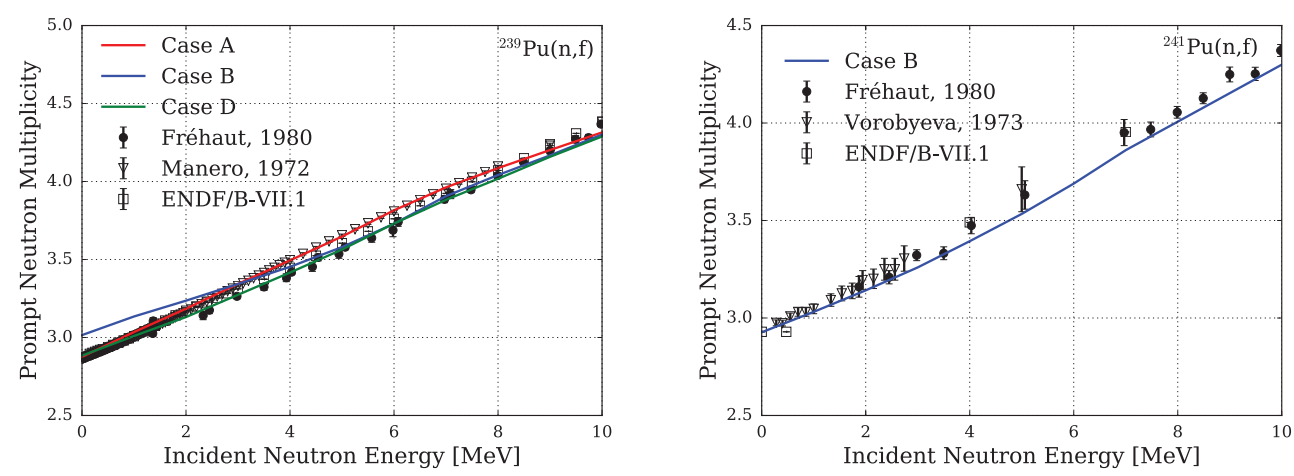

Figure 2. Comparison of the total prompt neutron multiplicity $\bar{v}$ (including pre-scission neutrons) from three data sources $[34,36,37]$ and three calculated cases. The cases correspond to using experimental $Y(A)[12](A)$, using calculated yields [13] and the same TKE parameters $(B)$, and using calculated yields and adjusting the TKE parameters $(D)$. We note that the change in TKE parameters is necessary in order to match the $\bar{v}\left(E_{n}\right)$ from experiment, but this produces strong tensions with $\langle\mathrm{TKE}\rangle$ data, as seen in Fig. 3. Only case $B$ is considered for ${ }^{241} \mathrm{Pu}(\mathrm{n}, \mathrm{f})$ and is compared to Ref. [34, 37, 38], with good agreement.

We can see that a simple swap of the $Y(A)$ results in a change in $\bar{v}$, when we require the $\langle$ TKE $\rangle$ to be equal in both cases $A$ and $B$. This is precisely caused by the difference in $\left\langle A_{h}\right\rangle$ between the experimental yields and the calculated ones. The shape of the $\langle\mathrm{TKE}\rangle(A)$ distributions are kept constant, but we must scale the magnitude by a factor $f$, such that $\langle$ TKE $\rangle$ are equal between cases $A$ and $B$. The calculated yields have a lower $\left\langle A_{h}\right\rangle$, so $f_{B}<f_{A}$. This shift to an overall lower $\langle\mathrm{TKE}\rangle(A)$ in case $B$ generates an overall larger total excitation energy via energy conservation and, thus, case $B$ shows a larger $\bar{v}$. The change in slopes between cases $A$ and $B$ reflect the differences in the evolution of $\left\langle A_{h}\right\rangle$ with incident neutron energy for the two yields methods.

Case $D$ is the result of altering both the $\langle\mathrm{TKE}\rangle$ and its slope in $E_{n}$ in order to match the shape of the experimental $\bar{v}$ [34]. We can see in Fig. 2 that these changes restore the agreement for ${ }^{239} \mathrm{Pu}(\mathrm{n}, \mathrm{f})$. This change in the $\langle\mathrm{TKE}\rangle$ slope acts to counter the difference generated from the change in $\left\langle A_{h}\right\rangle$ with $E_{n}$ between the experimental and calculated yields. The benefit of using a correlated fission event method, such as CGMF, is that the connection between $\langle\mathrm{TKE}\rangle$ and $\bar{v}$ is preserved. Thus, we can see the impact of this change in the TKE parameters in Fig. 3 and determine an uncertainty from the use of calculated yields. We find that $\delta_{\bar{v}} \sim 0.13 v / f, \delta_{\partial \bar{v} / \partial E_{n}} \sim 0.05 v / f / \mathrm{MeV}, \delta_{\langle\mathrm{TKE}\rangle} \sim$ $1.0 \mathrm{MeV}$, and $\delta_{\partial\langle\mathrm{TKE}\rangle / \partial E_{n}} \sim 0.18$. With an average energy cost of $\sim 8.0 \mathrm{MeV}$ per neutron, the values of $\delta_{\langle\mathrm{TKE}\rangle} \sim 1.0 \mathrm{MeV}$ are in agreement with the inferred $\delta_{\bar{v}} \sim 0.13 v / f$. The error bars for $\langle\mathrm{TKE}\rangle\left(E_{n}\right)$ are significantly larger in the ${ }^{241} \mathrm{Pu}$ case, where we find that the calculated yields can reproduce the $\langle\mathrm{TKE}\rangle$ and $\bar{v}$ data simultaneously. This is most likely due to the larger experimental error bars and, thus, a larger acceptable range for $\langle\mathrm{TKE}\rangle$, but may also indicate that this issue is a local problem instead of a global issue with the calculated yields. Further studies of uranium isotopes are underway to examine this.

The majority of this work has focused on a comparison of prompt neutron data, and its connection to the TKE of the fragments. We have also done a comparison of the prompt gamma observables, mainly the average total gamma energy $\left\langle E_{\gamma}^{T}\right\rangle$ and average total multiplicity $\left\langle M_{\gamma}\right\rangle$ as a function of incident neutron energy. These values are very sensitive to detector thresholds [40] and very limited 

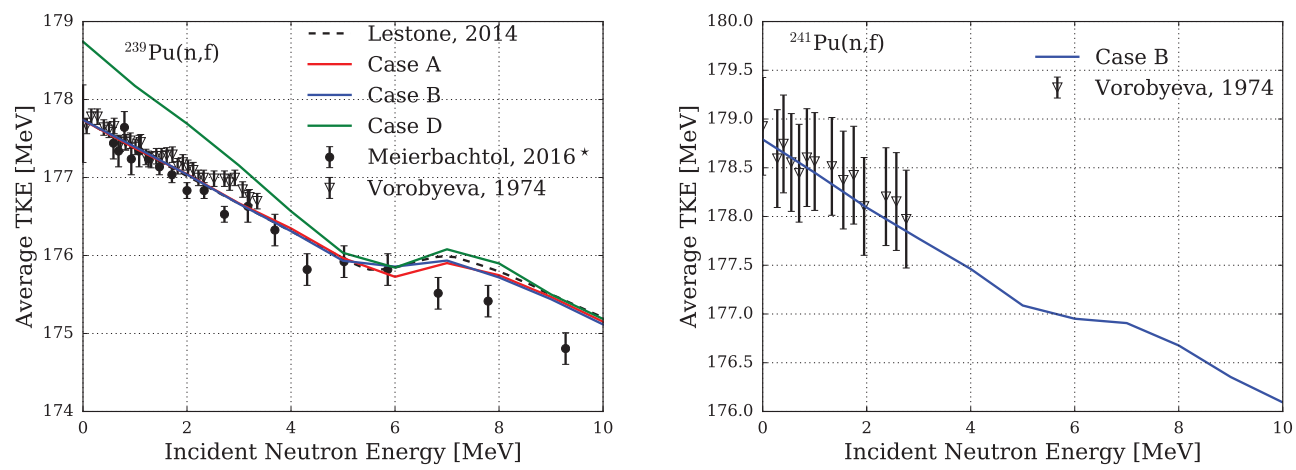

Figure 3. Comparison of the average total kinetic energy of the fission fragments $\langle\mathrm{TKE}\rangle$ from two data sources [19, 22], a parameterized model [39], and the CGMF calculations under the same case conditions as above. The data and parameterized model have been adjusted to have the same value for thermal neutrons. We note that the Meierbachtol data [22] corresponds to the post-neutron emitted kinetic energies. The shape beyond second chance fission implies a lower $\langle\mathrm{TKE}\rangle$ for the fissioning nucleus of ${ }^{239} \mathrm{Pu}$ than the one used in our simple $Z^{2} / A^{1 / 3}$ fit. One can clearly see that altering the TKE parameters to match the $\bar{v}\left(E_{n}\right)$ in Fig. 2 with the calculated yields results in an incompatible $\langle$ TKE $\rangle$ (case $D$ ). Again, only case $B$ is considered for ${ }^{241} \mathrm{Pu}(\mathrm{n}, \mathrm{f})$ and is compared to Ref. [23], finding good agreement, although the error bars are large.

data is available across our range of incident neutron energies. Nevertheless, we observe a very small impact of the yields choice on the $\left\langle E_{\gamma}^{T}\right\rangle$ and $\left\langle M_{\gamma}\right\rangle$. This is because any extra excitation energy induced through the changes in yields is dominantly exhausted through prompt neutron emission, especially at the early stages of the Hauser-Feshbach de-excitation. The resulting errors are $\delta_{\left\langle M_{\gamma}\right\rangle} \sim 0.01 \gamma / f$ and $\delta_{\left\langle E_{\gamma}^{T}\right\rangle} \sim 0.09 \mathrm{MeV}$ across the incident neutron energies studied here. This result indicates that these prompt $\gamma$-ray observables are less sensitive to the use of calculated versus experimental fission yields. More specific measurements, such as the intensity of low-energy $\gamma$-ray peaks, are more sensitive to the choice of $Y(A)$, on the order of $\sim 5 \%$. A more detailed analysis of changes to the $\gamma$-ray spectrum is underway.

\section{Conclusion}

We have successfully implemented theoretically-predicted yields from the macroscopic-microscopic model into a Hauser-Feshbach statistical decay formalism. This allows us to infer the feasibility of using theoretically-calculated mass yields to determine the prompt neutron and $\gamma$-ray observables for unknown fissioning systems. First, we presented a direct comparison of the mass yields for ${ }^{239} \mathrm{Pu}(\mathrm{n}, \mathrm{f})$ with available data. The average heavy fragment masses differed by $1.15 \mathrm{amu}$ and the change with $E_{n}$ in the symmetric region was more dramatic in the theoretical yields. These differences were propagated to the prompt neutron and $\gamma$-ray observables using the statistical Monte Carlo HauserFeshbach decay code CGMF.

The difference in $\left\langle A_{h}\right\rangle$ induced a difference in the $\langle\mathrm{TKE}\rangle$ between the experimental and theoretical mass yields. This, in turn, resulted in a difference in the prompt neutron multiplicity $\bar{v}$. We have demonstrated that the experimental data can simultaneously reproduce the $\bar{v}$ and $\langle\mathrm{TKE}\rangle$ trends with incident neutron energy. The calculated yields, however, introduce tension between these two observables. A resulting uncertainty of $\delta_{\bar{v}} \sim 0.13$ and $\delta_{\langle\mathrm{TKE}\rangle} \sim 1.0 \mathrm{MeV}$ for the thermal values and 
$\delta_{\partial \bar{v} / \partial E_{n}} \sim 0.05$ and $\delta_{\partial\langle\mathrm{TKE}\rangle / \partial E_{n}} \sim 0.18 \mathrm{MeV}$ for the slopes was determined for the calculated yields. These uncertainties are mainly derived from the ${ }^{239} \mathrm{Pu}(\mathrm{n}, \mathrm{f})$ reaction. Good simultaneous agreement between $\bar{v}$ and $\langle\mathrm{TKE}\rangle$ in the ${ }^{241} \mathrm{Pu}(\mathrm{n}, \mathrm{f})$ reaction was found when using the calculated yields. The uncertainties on the prompt $\gamma$-ray observables are much smaller: $\delta_{\left\langle M_{\gamma}\right\rangle} \sim 0.01$ and $\delta_{\left\langle E_{\gamma}^{T}\right\rangle} \sim 0.09 \mathrm{MeV}$ across the $10 \mathrm{MeV}$ range of incident neutron energies.

With these derived uncertainties, we identify the current applications of the theoretically calculated yields. In criticality studies, which often require uncertainties on $\bar{v}$ to be much less than $1 \%$ [35], calculations with theoretically calculated yields would be difficult and the more-precise experimental values must be used. However, one could use the calculated yields to estimate the contribution to a prompt neutron multiplicity distribution from low-level impurities in a sample during active or passive neutron interrogation. In addition, the derived uncertainties are very encouraging for fission calculations in r-process simulations [41-43]. For estimates of the $\gamma$-ray heating due to fission of fissiles without experimental data, we find that the induced error from these calculated yields are low. Additional studies will be conducted on more actinides and super-heavy nuclei to extend our analysis.

The authors would like to thank T. Kawano for useful discussions on the calculations of the multi-chance fission probabilities. The work of P.J., P.M., I.S., and P.T. was conducted under the auspices of the National Nuclear Security Administration of the U.S. Department of Energy at Los Alamos National Laboratory under Contract DEAC52-06NA25396. The work of C.S. acknowledges support through FUSTIPEN (French-U.S. Theory Institute for Physics with Exotic Nuclei) under DOE grant number DE-FG02-10ER41700. This publication is LA-UR-17-27821.

\section{References}

[1] M.G. Mustafa, P.A. Baisden, H. Chandra, Physical Review C 25, 2524 (1982)

[2] A.V. Karpov, P.N. Nadtochy, D.V. Vanin, G.D. Adeev, Physical Review C 63, 054610 (2001)

[3] P. Nadtochy, E. Ryabov, A. Gegechkori, Y.A. Anischenko, G. Adeev, Physical Review C 85, 064619 (2012)

[4] W. Younes, D. Gogny, Physical Review Letters 107, 132501 (2011)

[5] N. Schunck, D. Duke, H. Carr, A. Knoll, Physical Review C 90, 054305 (2014)

[6] A. Bulgac, P. Magierski, K.J. Roche, I. Stetcu, Physical Review Letters 116, 122504 (2016)

[7] J. Randrup, P. Möller, Physical Review Letters 106, 132503 (2011)

[8] T. Ichikawa, A. Iwamoto, P. Möller, A.J. Sierk, Physical Review C 86, 024610 (2012)

[9] Y. Aritomo, S. Chiba, K. Nishio, Progress in Nuclear Energy 85, 568 (2015)

[10] W. Hauser, H. Feshbach, Physical Review 87, 366 (1952)

[11] P. Talou, T. Kawano, I. Stetcu, LA-CC-13063, Los Alamos Nat. Lab. (2013)

[12] N. Akimov, V. Vorob'eva, V. Kabenin, Soviet Journal of Nuclear Physics-USSR 13, 484 (1971)

[13] P. Möller, T. Ichikawa, Eur. Phys. J. A 51, 173 (2015)

[14] P. Möller, C. Schmitt, Eur. Phys. J. A 53, 7 (2017)

[15] A.C. Wahl, Systematics of fission-product yields, Los Alamos Nat. Lab. (2002)

[16] H. Thierens, A. De Clercq, E. Jacobs, D. De Frenne, P. D’hondt, P. De Gelder, A. Deruytter, Physical Review C 23, 2104 (1981)

[17] C. Wagemans, E. Allaert, A. Deruytter, R. Barthélémy, P. Schillebeeckx, Physical Review C 30, 218 (1984)

[18] P. Schillebeeckx, C. Wagemans, A. Deruytter, R. Barthelemy, Nuclear Physics A 545, 623 (1992) 
[19] V. Vorobyeva, N. Dyachenko, N. Kolosov, B. Kuzminov, A. Sergachev, Soviet Journal of Nuclear Physics-USSR 19, 489 (1974)

[20] E. Allaert, C. Wagemans, G. Wegener-Penning, A. Deruytter, R. Barthélémy, Nuclear Physics A 380, 61 (1982)

[21] H. Thierens, E. Jacobs, P. D’hondt, A. De Clercq, M. Piessens, D. De Frenne, Physical Review C 29, 498 (1984)

[22] K. Meierbachtol, F. Tovesson, D. Duke, V. Geppert-Kleinrath, B. Manning, R. Meharchand, S. Mosby, D. Shields, Physical Review C 94, 034611 (2016)

[23] V. Vorobyeva, N. Dyachenko, N. Kolosov, B. Kuzminov, A. Sergachev, V. Surin, Soviet Journal of Nuclear Physics-USSR 19, 621 (1974)

[24] V. Vorobyeva et al., Mass yields and kinetic energy of fragments for fission of plutonium isotopes, in Conf. on Neutron Physics 3, 270 (1973)

[25] V. Surin, S. AI, B. Kuzminov, R. NI, Soviet Journal of Nuclear Physics-USSR 14, 523 (1972)

[26] K. Nishio, Y. Nakagome, I. Kanno, I. Kimura, Journal of Nuclear Science and Technology 32, 404 (1995)

[27] C. Tsuchiya, Y. Nakagome, H. Yamana, H. Moriyama, K. Nishio, I. Kanno, K. Shin, I. Kimura, Journal of Nuclear Science and Technology 37, 941 (2000)

[28] J. Neiler, F. Walter, H. Schmitt, Physical Review 149, 894 (1966)

[29] V. Viola, K. Kwiatkowski, M. Walker, Physical Review C 31, 1550 (1985)

[30] P. Talou, B. Becker, T. Kawano, M. Chadwick, Y. Danon, Physical Review C 83, 064612 (2011)

[31] B. Becker, P. Talou, T. Kawano, Y. Danon, I. Stetcu, Physical Review C 87, 014617 (2013)

[32] T. Kawano, Los Alamos National Laboratory, unpublished (2003)

[33] K. Shibata, O. Iwamoto, T. Nakagawa, N. Iwamoto, A. Ichihara, S. Kunieda, S. Chiba, K. Furutaka, N. Otuka, T. Ohsawa et al., Journal of Nuclear Science and Technology 48, 1 (2011)

[34] M. Chadwick, M. Herman, P. Obložinskỳ, M.E. Dunn, Y. Danon, A. Kahler, D.L. Smith, B. Pritychenko, G. Arbanas, R. Arcilla et al., Nuclear Data Sheets 112, 2887 (2011)

[35] R. Vogt, J. Randrup, J. Pruet, W. Younes, Physical Review C 80, 044611 (2009)

[36] F. Manero, V. Konshin, Atomic Energy Review 10, 637 (1972)

[37] M. Soleilhac, J. Frehaut, J. Gauriau, G. Mosinski, Data EXFOR 20 (1980)

[38] V. Vorobyeva, N. Dyachenko, B. Kuzminov, Soviet Journal of Nuclear Physics-USSR 36, 406 (1974)

[39] J. Lestone, T. Strother, Nuclear Data Sheets 118, 208 (2014)

[40] I. Stetcu, P. Talou, T. Kawano, M. Jandel, Physical Review C 90, 024617 (2014)

[41] I. Panov, E. Kolbe, B. Pfeiffer, T. Rauscher, K.L. Kratz, F.K. Thielemann, Nuclear Physics A 747, 633 (2005)

[42] G. Martínez-Pinedo, D. Mocelj, N.T. Zinner, A. Kelić, K. Langanke, I. Panov, B. Pfeiffer, T. Rauscher, K.H. Schmidt, F.K. Thielemann, Progress in Particle and Nuclear Physics 59, 199 (2007)

[43] S. Goriely, A. Bauswein, H.T. Janka, The Astrophysical Journal Letters 738, L32 (2011) 
